arXiv:hep-ph/0411060

\title{
The Ghost-Gluon Vertex in Landau Gauge Yang-Mills Theory*
}

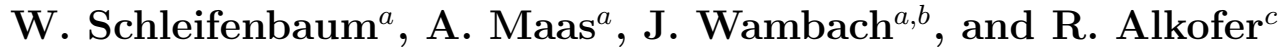 \\ ${ }^{a}$ Institute for Nuclear Physics, Technical University Darmstadt, \\ Schloßgartenstr. 9, D-64289 Darmstadt, Germany \\ ${ }^{b}$ Gesellschaft für Schwerionenforschung mbH, \\ Planckstr. 1, D-64291 Darmstadt, Germany \\ ${ }^{c}$ Institute for Theoretical Physics, Tübingen University, \\ Auf der Morgenstelle 14, D-72076 Tübingen, Germany \\ * Talk given by W.S. at the International School of Subnuclear Physics, \\ August 29 - September 07, 2004, in Erice, Italy.
}

\begin{abstract}
Possible mechanisms for gluon confinement in Yang-Mills theory in covariant gauges are discussed briefly. Implications for the infrared behavior of the Green's functions are outlined. Results for the gluon and Faddeev-Popov ghost propagators from DysonSchwinger studies and lattice calculations are presented. The importance of the ghostgluon vertex in these studies is discussed. Numerical results for a non-perturbatively constructed ghost-gluon vertex in four and three space-time dimensions are presented.
\end{abstract}

\section{Confinement in Covariant Gauges}

The strong interactions governing hadron and ultimately nuclear dynamics are described by quantum chromodynamics, the theory of quarks and gluons. This theory has several genuine non-perturbative features, such as e.g. dynamical chiral symmetry breaking. The most prominent phenomenon is, however, confinement. Strong evidence exists that it is generated in the Yang-Mills subsector, i.e. by the gluons. Naïvely, a Yang-Mills theory is defined in terms of the generating functional [1]

$$
\begin{aligned}
Z[J] & =\int \mathcal{D} A_{\mu}^{a} \exp \left(-\int d^{d} x \mathcal{L}+\int d^{d} x J_{\mu}^{a} A_{\mu}^{a}\right), \\
\mathcal{L} & =\frac{1}{4} F_{\mu \nu}^{a} F_{\mu \nu}^{a} \\
F_{\mu \nu}^{a} & =\partial_{\mu} A_{\nu}^{a}-\partial_{\nu} A_{\mu}^{a}+g_{d} f^{a b c} A_{\mu}^{b} A_{\nu}^{c} .
\end{aligned}
$$


Here we have already chosen a description in Euclidean space-time, which is used throughout this work. $A_{\mu}^{a}(x)$ is the gluon field, $F_{\mu \nu}^{a}(x)$ is the field strength tensor, $g_{d}$ is the gauge coupling constant in $d$ dimensions, and $f^{a b c}$ are the structure constants of the gauge group. The measure of the functional integral (11) extends over the complete configuration space of the gluon field.

However, the functional integral (11) is ill-defined. The Lagrangian is invariant under local gauge transformations and therefore the complete configuration space contains infinite sets of mutually equivalent field configurations. They are said to lie on the same gauge orbit. To avoid this over-counting in the functional integral, it is necessary to select only one copy on each gauge orbit. One tries to achieve this by gauge-fixing [1. Implementing the linear covariant gauge fixing condition $\partial^{\mu} A_{\mu}^{a}=0$ leads to the generating functional

$$
\begin{aligned}
Z[J] & =\int \mathcal{D} A_{\mu}^{a} M \exp \left(-\int d^{d} x\left(\mathcal{L}+\mathcal{L}_{\mathrm{gf}}\right)+\int d^{d} x J_{\mu}^{a} A_{\mu}^{a}\right), \\
M & =\operatorname{det}\left(-\partial_{\mu} D_{\mu}^{a b}\right) \\
D_{\mu}^{a b} & =\partial_{\mu} \delta^{a b}+g_{d} f^{a b c} A_{\mu}^{c},
\end{aligned}
$$

where $M$ is the Faddeev-Popov determinant, and $\mathcal{L}_{\text {gf }}$ the gauge-fixing term. The determinant is then rewritten as a functional integral over scalar Grassmann fields, the Faddeev-Popov ghosts. The generating functional then reads

$$
Z[J=0]=\int \mathcal{D} A_{\mu}^{a} \mathcal{D} \bar{c}^{b} \mathcal{D} c^{c} \exp \left(-\int d^{d} x\left(\mathcal{L}+\mathcal{L}_{\mathrm{gf}}+\bar{c}^{d} \partial_{\nu} D_{\nu}^{d e} c^{e}\right)\right) .
$$

The ghost and anti-ghost fields, $c^{a}(x)$ and $\bar{c}^{a}(x)$, respectively, encode part of the quantum fluctuations of the gluon field. Their appearance is a generic feature of covariant gauges. As will be detailed below, this procedure is in general not sufficient to completely specify the gauge beyond the use in perturbation theory.

One of the most intriguing features of QCD is confinement. Mechanisms for gluon confinement have been related to the infrared behaviour of propagators, see e.g. ref. [2]. We will focus here on aspects which have been substantiated recently, $c f$. section 2 .

First we discuss a sufficient but not necessary condition for confinement. If a propagator $D$ vanishes sufficiently fast in the infrared,

$$
\lim _{p^{2} \rightarrow 0} D\left(p^{2}\right)=0
$$

then the "particle" represented by $D$ cannot have a positive semi-definite spectral norm. Therefore it violates the Osterwalder-Schrader axiom of reflection positivity [3], and the propagator $D$ cannot have a Källen-Lehmann representation. Thus this "particle" is not part of the physical spectrum, and is hence confined [4].

For a massless excitation such as the gluon this confinement criterion is rather intuitive. Then, the condition (44) is just the statement that the on-shell propagator vanishes. Therefore, such a "particle" does not propagate, i.e. it is confined.

The criterion (4) is related to a scenario which was introduced by Kugo and Ojima [5]. In perturbation theory, ghosts and longitudinal gluons are not part of the physical spectrum, since they form doublets with respect to BRST charge. As these doublets are conjugate pairs by ghost number, this is called the quartet mechanism. The conjecture of Kugo and Ojima 
states that also transverse gluons form together with ghost-gluon bound states a quartet, and are thus not part of the physical spectrum. This does not imply that transverse gluons do not have asymptotic states. However, these states are not in the physical subspace and therefore do not contribute to $S$-matrix elements.

This scenario is based on a proof which requires three preconditions. The first is an unbroken BRST charge. Whether this is the case is not known. The second is the failure of cluster decomposition in the complete state space. This requires the existence of a massless excitation in the unphysical subsector. However, at the same time a mass gap in the physical subsector is required. The third precondition is an unbroken global color charge. This condition can be cast in Landau gauge into a condition for the ghost propagator $D_{G}[6]$

$$
\lim _{p^{2} \rightarrow 0} p^{2} D_{G}\left(p^{2}\right) \rightarrow \infty
$$

As will be seen below this scenario also entails condition (4) for the gluon propagator.

Another scenario has been proposed by Zwanziger [7] based on work by Gribov [8]. Only one gauge copy on each gauge orbit should be included in the functional integral constituting the generating functional. However, no local gauge condition is known which is able to specify the gauge completely also for large gauge field fluctuations. This is known as the Gribov problem [8]. The origin of this problem actually provides a possible handle to understand confinement. Therefore a closer look at the properties of the field configuration space is necessary.

It is possible to partition configuration space by the zeros of the Faddeev-Popov determinant (2). Concentric regions are found and each gauge orbit intersects each region at least once [8]. The region containing the origin, and thus relevant for perturbation theory, is known as the first Gribov horizon. It can be shown to be compact and convex. It has been found, that this region also contains gauge copies, see e.g. [9], and it is necessary to further restrict to the so-called fundamental modular region. This region is specified by minimizing an appropriate functional along each gauge orbit [10]. The fundamental modular region is also convex and compact around the origin [7.

The latter properties lead to an interesting observation. As configuration space is infinitedimensional, any volume is dominated by its boundary. It has therefore been argued that only the common boundary of the first Gribov horizon and the fundamental modular region contribute to any correlation function built from a finite number of field operators, especially Green's functions [7. Therefore, restricting to the first Gribov horizon, would be sufficient to calculate Green's functions.

This has a further important consequence [7]. The Faddeev-Popov determinant (2) vanishes by definition on this boundary. Therefore, the condition (5), termed in this context the Zwanziger horizon condition, is fulfilled as the determinant is directly linked to the inverse self-energy of the ghost. This also entails that the action is dominated by the gauge fixing term alone. Thus, in the extreme infrared all Green's functions are determined by contributions involving ghosts. This leads to the notion of ghost dominance.

Assuming the horizon condition (5), it can be shown that an infrared tree-level ghostgluon vertex complies with the renormalization group in leading order. This leads to the hypothesis of a bare ghost-gluon vertex also in the infrared. It has then been shown that, for the Landau gauge gluon propagator, the condition (4) follows and gluons are confined [7. 


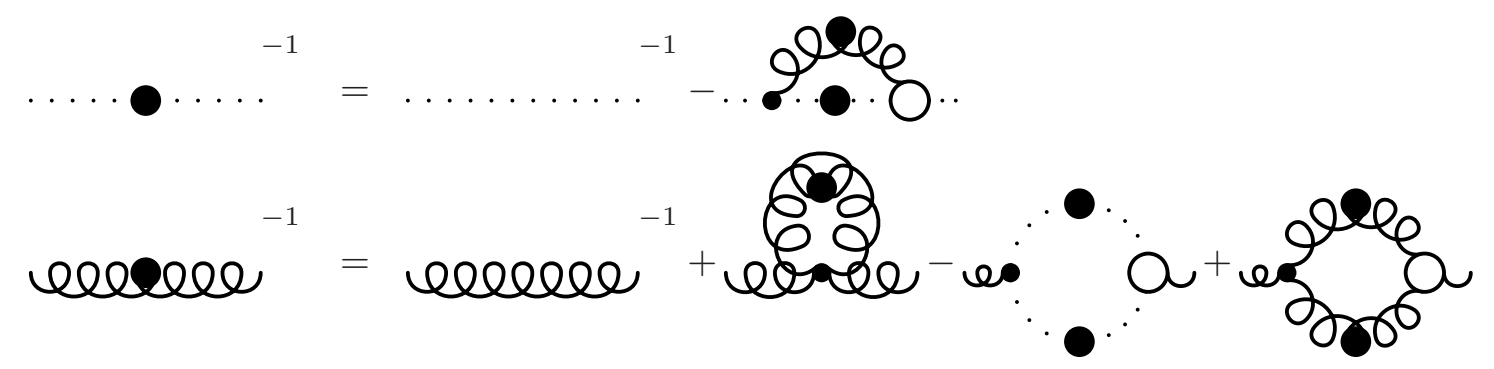

Figure 1: The Dyson-Schwinger equations for the 2-point functions of Yang-Mills theory. Dotted lines are ghosts and wiggly lines are gluons. Lines with a dot are full propagators, vertices with a small black dot are bare vertices and white circles are full vertices. The equation for the gluon has already been truncated to the one-loop level, see text.

Whether the identical infrared behavior of the ghost and gluon propagators within the Kugo-Ojima and the Zwanziger-Gribov scenarios, respectively, is more than a formal coincidence is not known. However, a ghost propagator with a stronger infrared divergence than a massless pole is also intuitively seen to be able to mediate confinement. After Fourier transformation such a singularity corresponds to a correlation being more long-range than that of a photon, and thus mediates a stronger force than a Coulomb force.

\section{Propagators in Yang-Mills Theory}

In both confinement scenarios described above the infrared behavior of propagators plays a central role. As infrared singularities are anticipated, $c f$. eq. (5), a non-perturbative method formulated in space-time continuum is desirable. Such a formulation is provided by the Dyson-Schwinger equations (DSEs), determining the Green's function of a theory, see e.g. [1, 2. A graphical representation of the equations for the propagators is shown in fig. 1.

The Landau gauge will be used throughout, as it is advantageous in the study of confinement for various reasons. First, the Landau gauge is a fixed point of the renormalization group. Second, the conditions for the Kugo-Ojima and Zwanziger-Gribov scenario take the simple forms (41) and (5) in Landau gauge. Last but not least, it is less singular than other gauges. This manifests itself in the bareness of the ghost-gluon vertex for a vanishing incoming ghost momentum [1]

$$
\lim _{p \rightarrow 0} \Gamma_{\mu}^{a b c}(k ; q, p)=i g_{d} f^{a b c} q_{\mu} .
$$

Here $p, q$, and $k$ are the momenta of the incoming ghost, the outgoing ghost, and the gluon, respectively.

Employing the ghost DSE and quite general assumptions, it can then be shown [12] that the ghost propagator necessarily fulfills condition (5). It is not possible in this generality to show that the gluon propagator satisfies (41). This requires a solution of the DSEs. However, it is not possible to solve these equations as they form an infinite set of coupled non-linear integral equations. Therefore, it is necessary to truncate the system.

During the last years, the truncation scheme depicted in fig. 11 has been established [13. This scheme has been successfully applied to Yang-Mills theory in four dimensions [14, 
three dimensions [15] and at finite temperature [16] as well as to QCD including dynamical quarks [17. In this scheme, all equations beyond those of the propagators are neglected, as well as genuine two-loop contributions in the equations for the propagators. Furthermore, a perturbative color structure of the Green's functions is assumed. It then only remains to specify the two remaining full vertices, the ghost-gluon and the 3-gluon vertex. The 3gluon vertex is chosen such that leading-order resummed perturbation theory is reproduced in the ultraviolet. Due to the property (6) the ghost-gluon vertex is usually taken to be bare. The results for the ghost and gluon propagators are shown in figs. 2 and 3 compared to lattice calculations. Both methods agree remarkably well. The largest deviations are found at intermediate momenta where the truncation scheme is expected to be least reliable. Although this scheme imposes several problems, especially concerning gauge invariance, in a systematic analysis only small quantitative effects due to truncation errors have been found, for a detailed discussion of this issue see e.g. refs. [14, 15].

Ghost dressing function in $4 \mathrm{~d}$

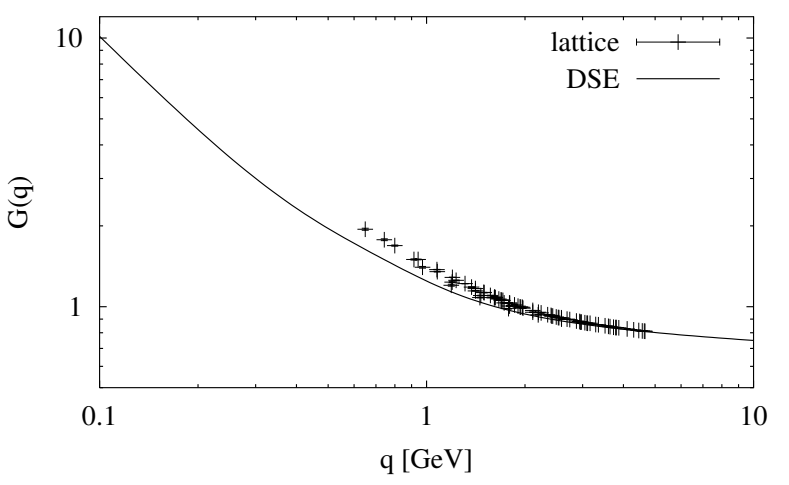

Ghost dressing function in 3d

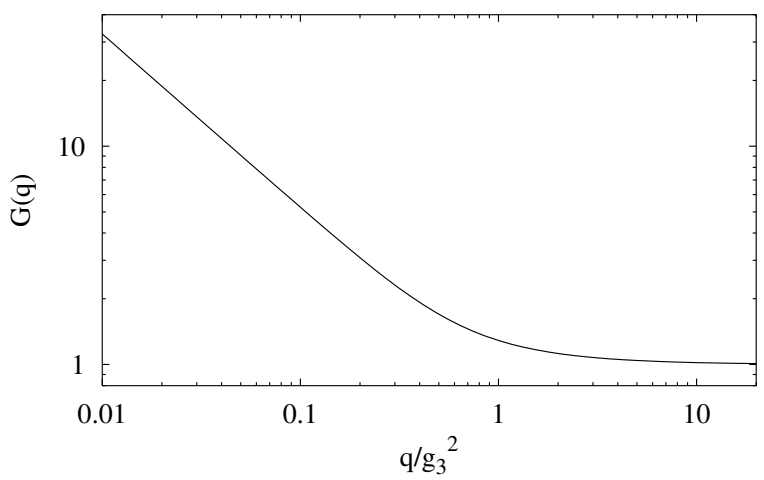

Figure 2: The ghost dressing function from DSEs in four dimensions 14] compared to lattice results [18] (left) and from DSEs in three dimensions [15] (right). $g_{3}$ is the dimensionful coupling constant in three-dimensional Yang-Mills theory.

The gluon propagator obeys condition (44), and the gluon is thus confined. As the ghost propagator also satisfies the Zwanziger horizon condition (15), the Kugo-Ojima and the Zwanziger-Gribov scenarios are both fulfilled. These findings have also been confirmed recently by exact renormalization group methods [22]. Furthermore, such propagators are in sharp contrast to the older concept of infrared slavery, which expected a gluon dressing function diverging as $1 / p^{2}[23]$. These earlier attempts neglected the ghost contribution 24. This so-called Mandelstam approximation leads to results contradicting current lattice results and can now be dismissed.

In the infrared, ghost dominance is found. Thus, besides the full gluon propagator, only diagrams with at least one ghost line in fig. 1 contribute. This is in accordance with the Zwanziger-Gribov scenario. The single crucial point in this derivation is then the structure of the ghost-gluon vertex [25]. It is therefore of great importance to understand it. The infrared behavior of the propagators has been studied using several ansätze, motivated by the Slavnov-Taylor identity (STIs) for the ghost-gluon vertex. The results are found to depend only quantitatively on the input vertex [25]. However, a more extensive analysis is highly desirable and will be presented below. 

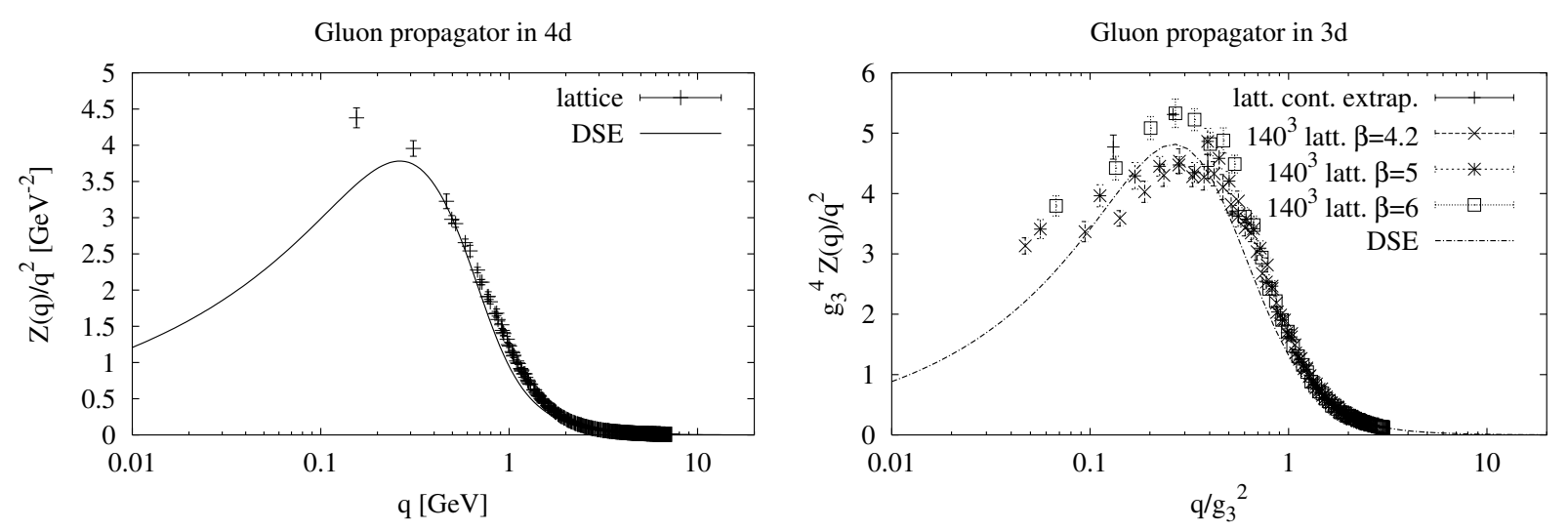

Figure 3: The gluon propagator from DSEs in four dimensions [14 compared to lattice data [19] (left) and from DSEs in three dimensions [15] compared to corresponding lattice data [20, 21] (right). Note that the lattice data at low momenta are likely to be strongly influenced by finite volume effects.

\section{Ghost-Gluon Vertex}

The most general tensor structure of the ghost-gluon vertex in Landau gauge is given by

$$
\Gamma_{\mu}^{a b c}(k ; q, p)=i g_{d}\left(q_{\mu}\left(f^{a b c}+A^{a b c}(k ; q, p)\right)+k_{\mu} B^{a b c}(k ; q, p)\right),
$$

where $A^{a b c}$ and $B^{a b c}$ are scalar functions. The tree-level contribution is left explicit. As there is no indication for a color structure different from perturbation theory [2], the structure constant is factored out, $A^{a b c}=: f^{a b c} A$ and $B^{a b c}=: f^{a b c} B$. Due to the transversality of the gluon propagator, the on-shell ghost-gluon vertex is transverse with respect to the gluon momentum. Therefore, the function $B$ is only relevant when not contracting with a transverse projector, e.g. in the corresponding STI. Hence, only the function $A$ will be discussed here. A detailed account on $B$ is given elsewhere [26].

In principle, the ghost-gluon vertex should be determined self-consistently by solving the DSEs for the propagators and the vertex simultaneously. A truncated DSE for the vertex neglecting four-point functions is shown in fig. 固. The completely self-consistent solution of such an equation is of significant technical complexity and has not been achieved yet. However, due to the hypothesis of Zwanziger, it is most likely sufficient to perform a semi-

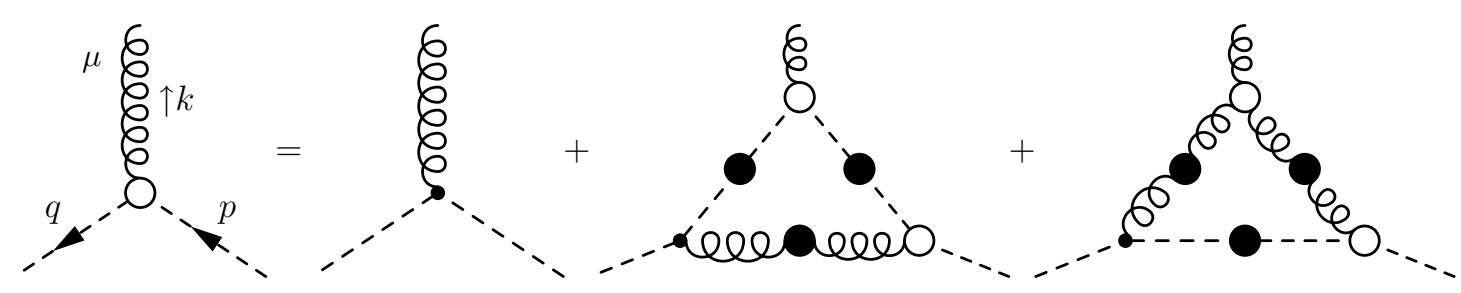

Figure 4: The DSE for the ghost-gluon vertex. Dotted lines are ghosts and wiggly lines are gluons. Lines with a dot are full propagators, vertices with a small black dot are bare vertices and white circles are full vertices. Contributions from the ghost-gluon scattering kernel have been removed, see text. 


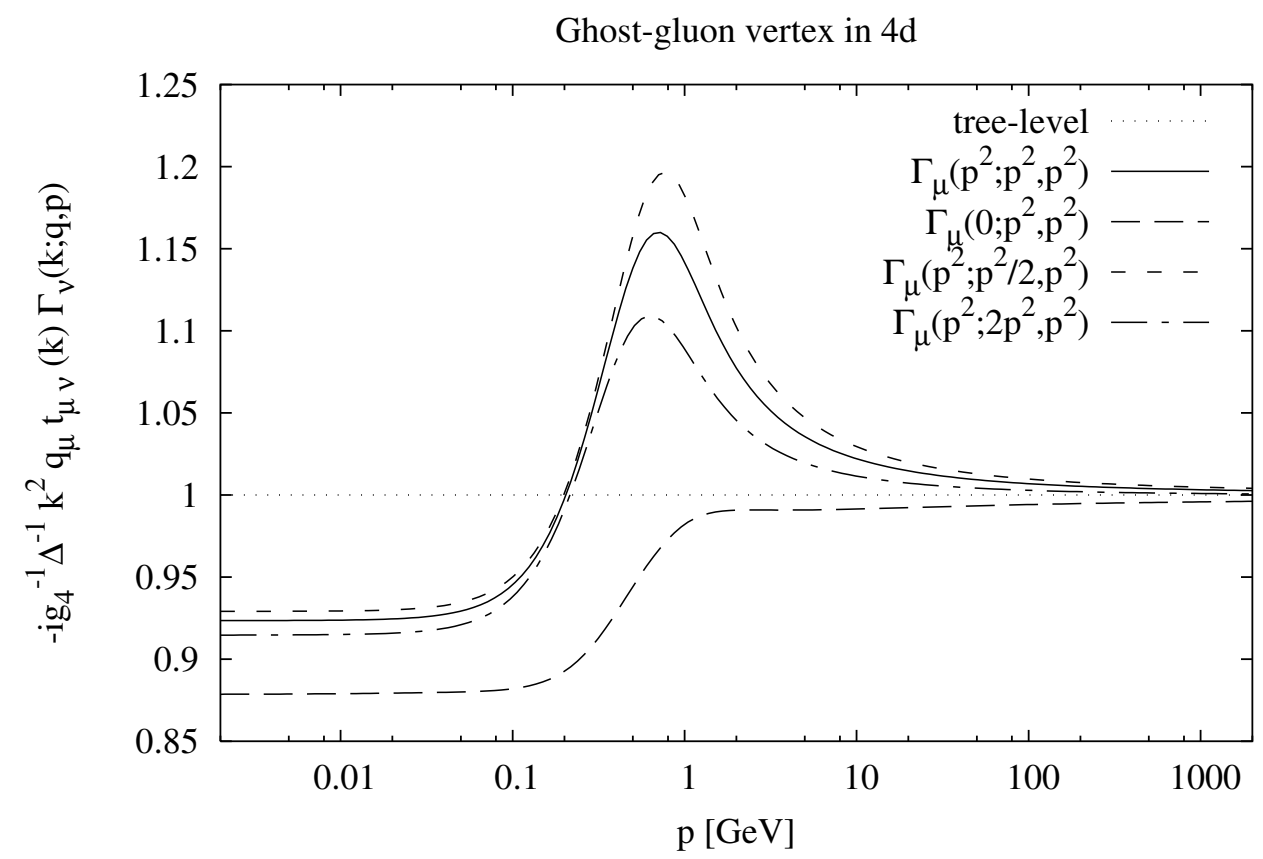

Figure 5: The normalized transverse part (8) of the ghost-gluon vertex in four dimensions in various kinematical regions.

perturbative calculation, i.e. to do one iteration step in the ghost-gluon vertex DSE. If the hypothesis is correct then the resulting vertex should not significantly deviate from the input tree-level vertex.

Hence, the evaluation scheme neglects the ghost-gluon scattering kernel in the ghostgluon vertex DSE. Further, as this is the first iteration step, all vertices are left bare and only the propagators are dressed with the results presented in the previous section. The results are displayed in fig. 5 for four dimensions and in fig. 6 for three dimensions. Shown is the transverse part of the ghost-gluon vertex, $1+A$, defined by

$$
\frac{k^{2}}{i g_{d} \Delta} q_{\nu}\left(\delta_{\mu \nu}-\frac{k_{\mu} k_{\nu}}{k^{2}}\right) \Gamma_{\mu}^{a b c}(k ; q, p)=f^{a b c}(1+A(k ; q, p))
$$

where $\Delta=q^{2} k^{2}-(q \cdot k)^{2}$ is a Gram determinant.

Several observations can be made [26. First, although not shown in the plots, the results comply with the infrared limit (6) and thus satisfy the respective STI in this specific kinematical limit. Second, they have the proper ghost-antighost symmetry [25], $A(k ; q, p)=$ $A(k ; p, q)$. Therefore, they fulfill all required conditions. In addition, in three dimensions, asymptotic freedom is manifest and the vertex reduces to the bare one in the ultraviolet. However, in four dimensions the anomalous dimensions is incorrect, as the vertex is not resummed yet.

As can be seen, the result is a vertex which is very close to the tree-level one at all momenta, especially in the infrared. This does not change in other kinematical conditions 26] and thus confirms Zwanziger's hypothesis in an impressive manner. The origin for the small deviations from the exact bare vertex in the infrared may be twofold. On the one hand, certainly iteration to self-consistency and truncation artifacts change the results 


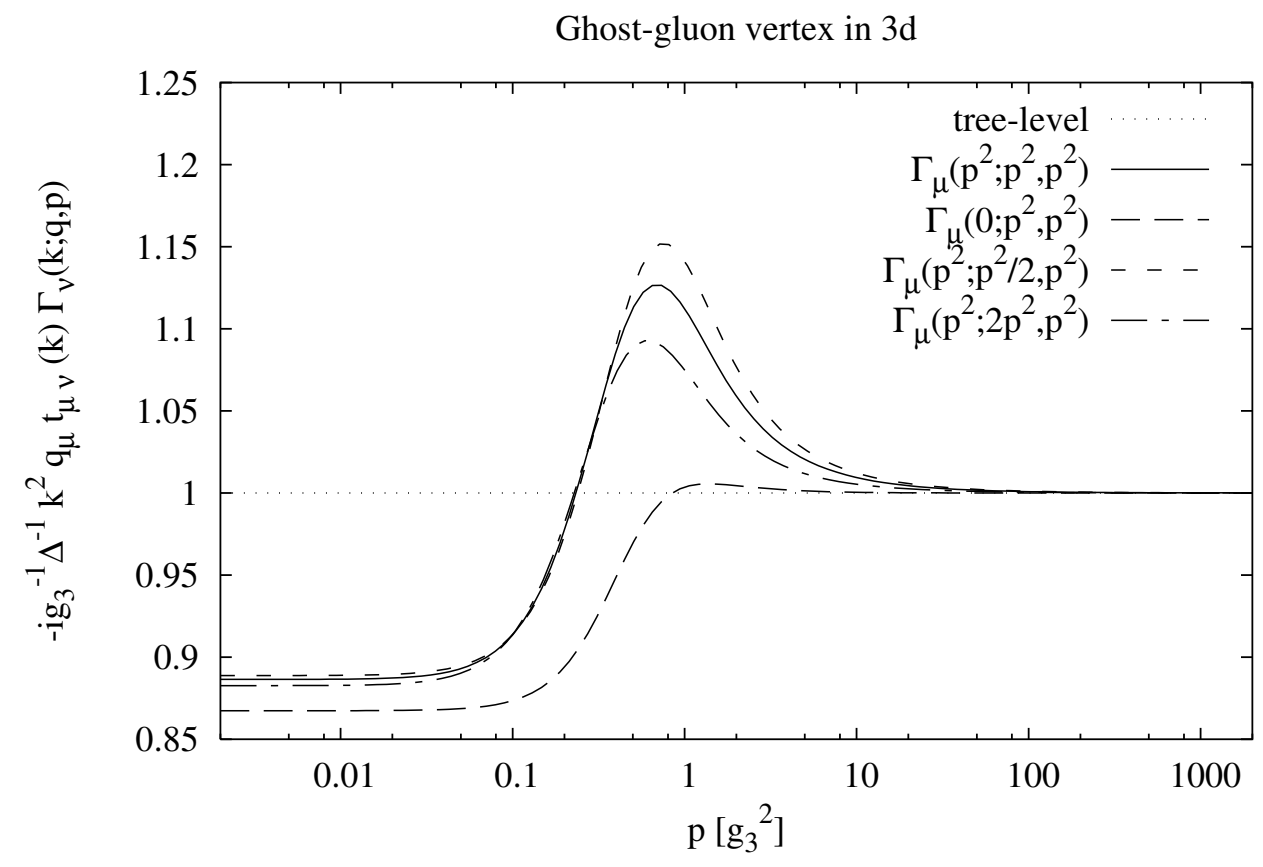

Figure 6: The normalized transverse part (8) of the ghost-gluon vertex in three dimensions in various kinematical regions.

quantitatively. As the ghost-gluon vertex DSE is already quite well fulfilled, this will most likely not lead to a qualitative change. On the other hand, in Zwanziger's hypothesis only agreement with leading-order renormalization group has been required. Subleading effects may generate a deviation of the order observed.

In addition, the vertex is nearly tree-level for all momenta. This supports the assumption of a bare ghost-gluon vertex in the truncation scheme for the propagators. It especially provides additional evidence for ghost dominance and therefore for the Zwanziger-Gribov confinement mechanism.

Similar results have also been reported recently in lattice calculations in four dimensions [27. They confirm the results found independently here and indicate also a nearly tree-level ghost-gluon vertex.

To assess the error of the approximations adopted, the full ghost-gluon vertices in the ansatz shown in fig. 4 have been replaced by the vertices motivated by the corresponding STI [25]. It has been found [26] that also these vertices are mapped to a nearly tree-level like vertex by the truncated DSE, as long as no singularity in an external momentum is introduced. (As there is no iteration in the current ansatz, these divergencies would survive the calculation.) Moreover, the results have been found to be slightly more sensitive to the propagators than to the input vertices. All these variations are quantitatively small. This indicates that the solution of the ghost-gluon vertex DSE is a vertex which is nearly tree-level.

It is probable that the influence of quarks, $c f$. ref. [17], or of an adjoint Higgs field, $c f$. ref. 15, on these findings is negligible. Neither couple directly to the ghost and therefore their presence does not give rise to new terms in the ghost-gluon vertex DSE. Hence, they can only contribute indirectly via their effect on the Yang-Mills propagators, which are very 
robust against coupling a small number of quark flavours [17, or scalars [15].

The ghost-gluon vertex may also be interesting with respect to extracting the running coupling constant [27. This issue has to be investigated further.

\section{Conclusions}

In Landau-gauge Yang-Mills theory we have calculated the ghost-gluon vertex employing non-perturbative propagators. The results show quite conclusively that deviations from the tree-level value are very small, even in the infrared. On the one hand, this supports the truncation scheme used to calculate the propagators of the Yang-Mills theory. The results found in these calculations give strong evidence for a confinement mechanism of the Kugo-Ojima or Zwanziger-Gribov type. Especially, no sign of an infrared enhanced gluon propagator in Landau gauge is found. More importantly, the results on the ghost-gluon vertex alone, provide independent support for the Zwanziger-Gribov scenario as they fulfill Zwanziger's hypothesis of a bare ghost-gluon vertex in the infrared. The results presented here are thus a further indication for a confinement mechanism driven by the dynamics on or near the Gribov horizon. This coincides with recent findings in Coulomb gauge [28. All these evidences combined promise a new era of quantitative confinement physics.

\section{Acknowledgments}

W. S. thanks the organizers of the International School of Subnuclear Physics for the very interesting meeting and for the opportunity to present this talk. We are grateful to C. S. Fischer, J. M. Pawlowski and L. von Smekal for helpful discussions. This work is supported by the BMBF under grant numbers 06DA917 and 06DA116, and by the Helmholtz association (Virtual Theory Institute VH-VI-041).

\section{References}

[1] R. J. Rivers, "Path Integral Methods In Quantum Field Theory," Cambridge, UK: Univ. Pr. (1987) 339 p. (Cambridge monographs on mathematical physics).

[2] R. Alkofer and L. von Smekal, Phys. Rept. 353 (2001) 281 arXiv:hep-ph/0007355 and references therein.

[3] K. Osterwalder and R. Schrader, Commun. Math. Phys. 31 (1973) 83. K. Osterwalder and R. Schrader, Commun. Math. Phys. 42 (1975) 281.

[4] R. Oehme and W. Zimmermann, Phys. Rev. D 21 (1980) 1661. R. Oehme and W. Zimmermann, Phys. Rev. D 21 (1980) 471.

[5] T. Kugo and I. Ojima, Prog. Theor. Phys. Suppl. 66 (1979) 1.

[6] T. Kugo, arXiv:hep-th/9511033.

[7] D. Zwanziger, Phys. Rev. D 69 (2004) 016002 arXiv:hep-ph/0303028 and references therein. 
[8] V. N. Gribov, Nucl. Phys. B 139 (1978) 1.

[9] P. van Baal, arXiv:hep-th/9711070

[10] D. Zwanziger, Nucl. Phys. B 412 (1994) 657.

[11] J. C. Taylor, Nucl. Phys. B 33 (1971) 436. W. J. Marciano and H. Pagels, Phys. Rept. 36 (1978) 137.

[12] P. Watson and R. Alkofer, Phys. Rev. Lett. 86 (2001) 5239 arXiv:hep-ph/0102332;

R. Alkofer, L. von Smekal and P. Watson, arXiv:hep-ph/0105142.

[13] L. von Smekal, R. Alkofer and A. Hauck, Phys. Rev. Lett. 79 (1997) 3591 arXiv:hep-ph/9705242 . L. von Smekal, A. Hauck and R. Alkofer, Annals Phys. 267 (1998) 1 [Erratum-ibid. 269 (1998) 182] arXiv:hep-ph/9707327.

[14] C. S. Fischer and R. Alkofer, Phys. Lett. B536 (2002) 177 arXiv:hep-ph/0202202;

[15] A. Maas, J. Wambach, B. Grüter and R. Alkofer, Eur. Phys. J. C37 (2004) 335 arXiv:hep-ph/0408074.

[16] B. Grüter, R. Alkofer, A. Maas and J. Wambach, arXiv:hep-ph/0408282

[17] C. S. Fischer and R. Alkofer, Phys. Rev. D 67 (2003) 094020 arXiv:hep-ph/0301094.

[18] K. Langfeld, J. C. R. Bloch, J. Gattnar, H. Reinhardt, A. Cucchieri and T. Mendes, arXiv:hep-th/0209173.

[19] P. O. Bowman, U. M. Heller, D. B. Leinweber, M. B. Parappilly and A. G. Williams, arXiv:hep-lat/0402032.

[20] A. Cucchieri, F. Karsch and P. Petreczky, Phys. Rev. D 64 (2001) 036001 arXiv:hep-lat/0103009.

[21] A. Cucchieri, T. Mendes and A. R. Taurines, Phys. Rev. D 67 (2003) 091502 arXiv:hep-lat/0302022.

[22] J. M. Pawlowski, D. F. Litim, S. Nedelko and L. von Smekal, Phys. Rev. Lett. 93 (2004) 152002 arXiv:hep-th/0312324; C. S. Fischer and H. Gies, arXiv:hep-ph/0408089.

[23] N. Brown and M. R. Pennington, Phys. Rev. D 38 (1988) 2266.

[24] S. Mandelstam, Phys. Rev. D 20 (1979) 3223.

[25] C. Lerche and L. von Smekal, Phys. Rev. D 65 (2002) 125006 arXiv:hep-ph/0202194.

[26] W. Schleifenbaum, A. Maas, J. Wambach and R. Alkofer, arXiv:hep-ph/0411052; W. Schleifenbaum, diploma thesis, TU Darmstadt, 2004.

[27] A. Cucchieri, T. Mendes and A. Mihara, arXiv:hep-lat/0408034.

[28] J. Greensite, S. Olejnik and D. Zwanziger, arXiv:hep-lat/0407032 